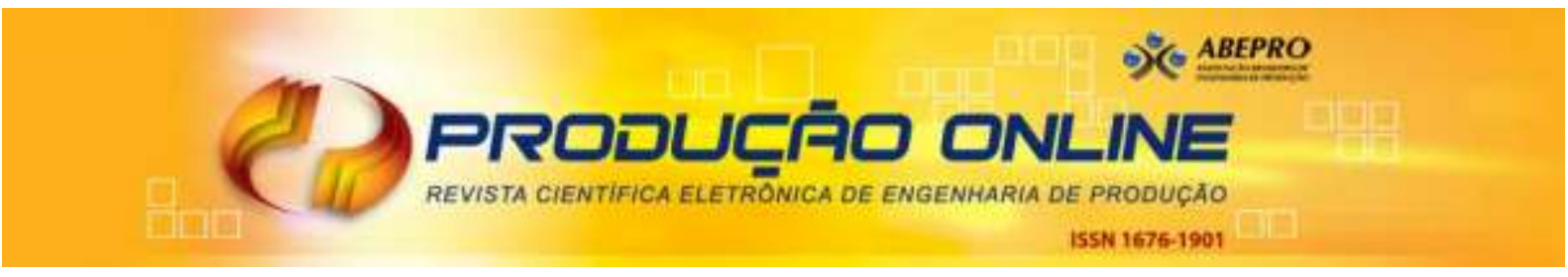

\title{
UMA ANÁLISE DO SISTEMA DE GESTÃO WMS: UM ESTUDO MULTICASO EM EMPRESAS DESENVOLVEDORAS E USUÁRIAS
}

\section{THE ANALYSIS OF WMS MANAGEMENT SYSTEM: A MULTI CASE STUDY IN DEVELOPER COMPANIES AND USERS}

\author{
Felipe Furlan Soriano*E-mail: fsoriano@usp.br \\ Alexandre Pereira Salgado Junior*E-mail: asalgado@usp.br \\ *Universidade de São Paulo (USP), Ribeirão Preto - SP
}

Resumo: É crescente o número de empresas que utilizam o WMS na operação de armazenagem, entretanto, boa parte das organizações que adotam este sistema tem dificuldades no processo de implantação ou em usufruir plenamente dos seus recursos. Neste contexto, a presente pesquisa tem por objetivo analisar o sistema WMS, identificando as principais barreiras inerentes ao seu processo de implantação, os benefícios e as funcionalidades atualmente oferecidos ao mercado, bem como as tendências para o aprimoramento da ferramenta. Para tanto, foi utilizado o método de estudo multicaso que abordou duas empresas desenvolvedoras do software e de três organizações usuárias e, a partir da análise de conteúdo das informações provenientes da revisão de literatura, das entrevistas semiestruturadas e da análise documental realizadas, foi possível identificar como principais dificuldades no processo de implantação do sistema as falhas no cadastro de produtos e endereços, o mapeamento dos processos logísticos, o treinamento de pessoal e a mudança cultural de trabalho. Quanto aos benefícios do sistema, salienta-se o melhor controle operacional e o incremento da acuracidade de estoque. Por fim, identificou-se que as tendências para o desenvolvimento da ferramenta envolvem a vinculação do sistema a tablets e smartphones, integração com a tecnologia RFID e geração de relatórios customizados.

Palavras-chave: Logística. Gestão da armazenagem. Controle operacional. Sistema de informação. WMS

Abstract: In recent years the number of companies that use WMS on its storage operation has increased. However, a great part of organizations that adopt this system has difficulties in the implementation process or in fully exploit its resources. In this context, this research aims to analyze the WMS system, identifying the key barriers inherent in its implementation process, the benefits and features curently offered to the market, as well as the trends to the tool development. This analysis was performed through the method of multicase study under the perspective of two system developers and three users. Based on the content analysis of the informations from literature review, the semistructered interviews and the documentary analysis performed was possible to identify as the main difficulties in the process of system implementation the flaws in registration of products and addresses, mapping of logistics processes, the staff training and cultural work change. Regarding the system benefits, stands out the better operational control and the stock accuracy improvement. Finally the trends for the tool improvement involve linking the system with tablets and smartphones, integrations with RFID technology and customized reports generation.

Keywords: Logistics. Storage Management. Operation Control. Information System. WMS

Revista Produção Online, Florianópolis, SC, v.14, n. 1, p. 195-218, jan./mar. 2014. 


\section{INTRODUÇÃO}

Com um consumidor cada vez mais exigente, o aumento da pressão por melhores preços e prazos de entrega tem sido uma constante, envolvendo rápidas variações de demanda, menores e mais frequentes pedidos, elevada competição e reinvindicações por melhores níveis de serviço (ROODBERGEN e VIS, 2009; CHIANG, LIN e CHEN, 2011).

Tal cenário implica em maior eficiência das organizações em suas atividades operacionais (GU, GOETSCHALCKX e MCGINNIS, 2007). Segundo Freitas et al (2010), o mercado vê-se forçado a aprimorar a sua competência logística, otimizando os seus processos tanto na execução do transporte quanto na gestão da armazenagem. Esta última, auxilia as empresas na redução dos custos e melhoria da produtividade nos armazéns.

Por estes motivos, vem sendo vista não mais como uma área de suporte, mas sim como um fator de significativa relevância em termos competitivos (KOSTER; LE-DUC e ROODBERGEN, 2007).

É, portanto, uma variável crítica para que as organizações tenham condições de proporcionar ao cliente um melhor nível de serviço (BAKER e CARNESSA, 2009; KUTANOGLU e LOHIYA, 2007; CHIEN MING et al., 2010; GAMBERINI et al., 2008; CHEN, LANGEVIN e RIOPEL, 2011), tornando-as individualmente mais eficientes e contribuindo em melhorias também à cadeia de suprimentos da qual fazem parte (ANG, LIM e SIM, 2012).

Para tanto, uma solução frequentemente encontrada é a implantação do sistema de gestão da armazenagem, o Warehouse Management System (WMS), que tem como finalidade a melhor utilização dos recursos inerentes à área de armazenagem, desde o espaço físico até a mão-de-obra disponível (FLEURY, WANKE e FIGUEIREDO, 2000).

Contudo, mesmo sendo uma ferramenta que contribui significativamente para a melhoria da eficiência logística, muitas empresas enfrentam dificuldades no processo de implantação do sistema e frequentemente não conseguem utilizar todos os recursos oferecidos, minimizando, portanto, os benefícios obtidos. Tal situação deve-se ao fato de que as organizações usuárias não conhecem todas as 
características e potencialidades da ferramenta ou não compreendem de fato a realidade do seu atual processo de armazenagem (BANZATO et al, 2010).

No intuito de contribuir com soluções, o presente artigo pretende analisar o sistema WMS, identificando as barreiras encontradas em seu processo de implantação, os benefícios e as funcionalidades atualmente oferecidos ao mercado, assim como as tendências para o aprimoramento da ferramenta, levando em consideração a visão das empresas consultadas.

Para tanto, foi aplicado o método de estudo multicasos envolvendo cinco empresas subdivididas em duas categorias: duas organizações desenvolvedoras do software de gestão e três empresas usuárias do sistema.

Realizadas as considerações iniciais, a seguir a pesquisa é descrita em três partes: a apresentação dos conceitos teóricos relevantes ao estudo, a descrição dos procedimentos metodológicos adotados e a explanação acerca dos resultados obtidos.

\section{REFERENCIAL TEÓRICO}

\subsection{Gestão da Cadeia de Suprimentos (SCM)}

O consumidor final acostumou-se a adquirir determinados produtos pelo bom nível de serviço prestado pela empresa fornecedora (VIJAYASARATHY, 2010). Entretanto, sabe-se que esta eficiência no atendimento é obtida a partir da combinação entre bom desempenho operacional do fornecedor e da cadeia de suprimentos da qual ele faz parte.

Esta constatação faz com que a gestão de operações não seja mais abordada do ponto de vista individual das empresas, atualmente esta questão envolve também as organizações participantes da cadeia de suprimentos (TRKMAN et al 2010).

Assim, compreende-se que "o SCM ajuda as empresas a gerir o fluxo de informação, dinheiro e produtos além das fronteiras físicas da organização" (SHIH et al., 2012, p. 70). Portanto, segundo Rutner, Gibson e Willims (2003), o processo logístico torna-se mais complexo por envolver duas ou mais empresas e passa a 
significar um procedimento crítico para que a cadeia seja mais competitiva e tenha condições de se destacar no mercado (ZHOU e BENTON, 2007).

O aprimoramento desta logística envolve, entre outros fatores, a questão da gestão dos estoques (BOWERSOX; CLOSS e COOPER, 2007; SOUZA; KLIEMANN NETO e ANZANELLO, 2012), o que demanda novas estratégias de gestão da armazenagem que sejam aplicáveis e gerem resultados à cadeia de suprimentos como um todo (MADADI, KURZ e ASHYERI, 2009).

Estes resultados positivos são provenientes da melhor utilização dos recursos disponíveis e do compartilhamento de informações entre clientes e fornecedores, fatores que aprimoram o processo logístico (YAO e DRASNER, 2008).

Portanto, compreende-se a relevância de que a operação de armazenagem, assim como as demais atividades logísticas, ocorram de forma eficiente, otimizando o desempenho da empresa e, consequentemente, da cadeia de suprimentos, o que agrega maior satisfação ao cliente final.

\subsection{Gestão da Armazenagem}

Neste cenário, pode-se afirmar que a gestão da armazenagem corresponde a uma das principais atividades executadas pela logística, compreendida como a gestão do espaço físico necessário para manter um estoque de mercadorias, englobando o arranjo físico (layout) e o controle da operação (RODRIGUES, 2011). Esta área é também responsável pela gestão da área de picking e realização do endereçamento dos produtos, minimizando o tempo de procura e movimentação tanto no recebimento como na expedição dos materiais (ACCORSI, MAZINI e BORTOLINI, 2012).

Um fator que agrega maior complexidade a estas operações é o aumento contínuo do número de itens em estoque, stock keeping units (SKUs), em virtude das variações das características físicas do produto, como o tamanho das embalagens ou ainda a diversificação de modelos, cores e sabores. Evidentemente, quanto maior a variedade dos itens, maior a necessidade de espaço e/ou melhor utilização e organização dos recursos disponíveis (GU, GOETSCHALCKX e MACGINNIS, 2007). 
Este contexto reflete direta e negativamente na produtividade do processo de separação de pedidos, atividade considerada por diversos autores no âmbito acadêmico como a mais onerosa do processo de armazenagem (CHIANG, LIN e CHEN, 2011; CHIEN-MING et al, 2010, ACCORSI, MANZINI e BORTOLINI, 2012; YU e KOSTER, 2010). Isso ocorre pois, conforme afirmado por Yu e Koster (2010, p. 1), "os colaboradores que realizam a apanha dos itens demandados na ordem de separação dispendem muito tempo procurando os produtos e movimentando-se para segregá-los".

Entretanto, as dificuldades descritas podem ser superadas por uma gestão eficiente já que, segundo Ballou (2006), a armazenagem executada e controlada de forma adequada contribui com a redução dos custos de transporte e produção; coordenação da oferta e demanda; suporte ao processo de produção; e colaboração com o processo de comercialização, minimizando os custos operacionais e maximizando a lucratividade da empresa (SAHIN e DALLERY, 2009; PONTES, PALMA e PORTO, 2008).

Portanto, fica claro que a otimização do processo de armazenagem gera reflexos positivos em diversas áreas da empresa, para a cadeia de suprimentos e para o cliente final. Porém, para que este cenário seja real, é necessária a aplicação de tecnologia na operação.

\subsection{TI aplicada à gestão da armazenagem}

A tecnologia é ferramenta primordial para a redução dos custos envolvidos no processo de armazenagem. Segundo Alves (2000, p. 154), "cada vez mais as empresas buscam na Tecnologia da Informação (TI) alternativas para facilitar o gerenciamento de suas atividades, visando aumentar o controle e obter informações precisas", o que ocorre em virtude da própria necessidade do mercado de aprimorar a sua produtividade e consequentemente o nível de serviço oferecido ao cliente (SALGADO JUNIOR et al., 2010; REGGIANI e DALCOL, 2011).

Evidentemente as organizações que usam tecnologias mais desenvolvidas do que as dos concorrentes têm condições de integrar, executar e controlar de forma mais eficiente as operações logísticas da cadeia de suprimentos (SANDERS e 
PREMUS, 2002). Portanto, estas empresas, independentemente do seu porte (PICK, DIESEL e SELLITO, 2011), podem obter melhor controle e uso de informações como suporte aos gestores na tomada de decisão, ficando propensas a realizar melhor as atividades operacionais (DIAS et al., 2007; TSEGN, WU e NGUYEN, 2011).

Este comportamento reflete-se no aumento do investimento das organizações em softwares que propiciem o aprimoramento dos processos logísticos (LOEBBECKE, POWELL, 1998). Dentre as opções de sistemas que envolvem o aperfeiçoamento da gestão da armazenagem, Dinter, Lahrmann e Winter (2012) destacam o Business Inteligence (BI) e o Warehouse Management System (WMS), o software mais utilizado para a otimização das atividades relacionadas à armazenagem (LUDWIG e GOOMAS, 2008) e que pede mais esclarecimentos.

\subsection{Warehouse Management System (WMS)}

O WMS pode ser definido, segundo Rodrigues (2011), como a integração entre hardware, software e equipamentos periféricos para gerenciar o estoque, o espaço físico, os equipamentos utilizados na movimentação interna, bem como auxiliar os colaboradores que nesta área atuam. Esta ferramenta de gestão otimiza as atividades operacionais (fluxo de materiais) e também as administrativas (fluxo de informação), atuando sobre o recebimento, a estocagem, a separação de pedidos, a expedição e o inventário (BANZATO, 2005).

Quando bem utilizado, o WMS pode proporcionar à empresa usuária três principais benefícios: o aumento da precisão das informações do estoque, a ampliação da velocidade e qualidade das operações do armazém e o incremento da produtividade do pessoal e dos equipamentos envolvidos neste processo (COSTA e GOOBO JUNIOR, 2008).

A dinâmica do sistema envolve o registro de cada movimentação por meio de coletores de código de barras - os dados são encaminhados em tempo real ao software, o que permite o monitoramento contínuo da operação, possibilitando maior agilidade na tomada de decisão e minimizando a ineficiência. 
A partir de tal controle, as empresas usuárias passam a ter uma melhor visibilidade do estoque, da capacidade de aprimoramento de sua acuracidade e também do processo de separação de pedidos, agregando mais velocidade no processo de expedição, além de otimizar a gestão das atividades operacionais (VAIDYANATHAN e DEVAJI, 2008).

Além disso, o sistema também monitora os itens em estoque, saber exatamente o que existe fisicamente no armazém por meio dos dados informados pelo WMS é o que define a precisão do controle da operação (SHIN e DALLERY, 2009). Este indicador é denominado acuracidade: quanto maior este índice, menor é a divergência entre o que consta no estoque físico e no estoque registrado no sistema.

Entretanto, existem algumas dificuldades na implantação do sistema, como o alto montante de investimento inicial, baixo conhecimento dos colaboradores sobre a ferramenta e a resistência à mudança, necessidade de remodelagem e adaptação dos processos operacionais e a integração entre hardware, software e os funcionários (BANZATO et al, 2010).

A partir da superação destas barreiras, o sistema pode auxiliar a empresa na redução dos seus custos operacionais e na melhoria do nível de serviço prestado. Neste sentido, Banzato (2005) destaca entre os principais benefícios oferecidos pelo WMS: a melhoria da acuracidade de inventário; melhoria da ocupação do espaço (endereçamento automático); redução de erros operacionais; aumento da produtividade; redução do tempo de pedido em espera e otimização do percurso de separação de pedidos.

Finalizadas as considerações sobre o referencial teórico que dá suporte à compreensão deste artigo, a seção a seguir versa sobre os procedimentos metodológicos adotados.

\section{MÉTODO DE PESQUISA}

O presente estudo pode ser caracterizado como uma pesquisa descritiva aplicada, pois retrata e analisa fatos já ocorridos nas organizações participantes deste trabalho acadêmico - "os fatos são observados, registrados, analisados, 
classificados e interpretados, sem que o pesquisador interfira sobre eles" (ANDRADE, 2004, p. 32). Segundo Oliveira Neto (2008), ela analisa fenômenos atuais de forma a gerar contribuições significativas para a resolução de problemas que ocorrem na realidade, alinhando-se, portanto, ao objetivo deste artigo.

Ainda sobre a tipologia, a pesquisa tem caráter predominantemente qualitativo, de forma que as informações analisadas são provenientes da aplicação de três técnicas de coleta de dados: a entrevista semiestruturada, observação nãoparticipante e análise documental. O uso de tais técnicas e de múltiplas fontes de evidência foi definido em virtude da convicção de que a qualidade das informações é mais importante do que o número de entrevistados ou ainda o número de estudos de caso (RICHARDSON, 1999).

As técnicas foram aplicadas em um estudo multicaso (YIN, 2009) realizado com cinco empresas definidas por conveniência, de acordo com a possibilidade de disponibilização de informações em profundidade suficiente para que os objetivos iniciais propostos pudessem ser atingidos. As empresas foram subdividas em duas categorias: duas desenvolvedoras do software WMS e três usuárias do sistema.

Foram realizadas visitas com durações variadas, de forma a não prejudicar as atividades diárias dos entrevistados, considerados "pessoas-chave": três analistas de logística sênior, um gerente de logística e dois coordenadores responsáveis pelo desenvolvimento do software WMS.

A coleta de dados teve por finalidade responder a quatro perguntas de pesquisa: (1) Quais são as novas funcionalidades oferecidas pelos desenvolvedores do WMS?; (2) Quais são as dificuldades encontradas no processo de implantação do WMS, as adaptações necessárias e os benefícios percebidos na visão tanto dos desenvolvedores como dos usuários da ferramenta?; (3) Quais são as tendências de aprimoramento para o WMS?

A fim de direcionar a análise das informações no sentido de responder às questões acima, foram definidas diferentes abordagens de pesquisa para cada categoria de empresas. Quanto aos desenvolvedores do software, buscou-se identificar as funcionalidades atualmente oferecidas ao mercado, bem como as suas considerações sobre as boas práticas em relação à implantação do WMS. Já para 
os usuários do sistema, a análise foi desenvolvida sobre as variáveis descritas a seguir:

Quadro 1 - Resumo das variáveis analisadas nos usuários do WMS

\begin{tabular}{|c|c|}
\hline Indicador & Variável \\
\hline \multirow{4}{*}{ Gestão de pessoas } & Conhecimento do conceito WMS antes da implantação \\
\hline & Preparação e treinamento pré-implantação do WMS \\
\hline & Mapeamento e alinhamento dos processos organizacionais \\
\hline & Apoio da alta gerência \\
\hline \multirow{2}{*}{$\begin{array}{l}\text { Tecnologia da } \\
\text { Informação }\end{array}$} & Adequação às necessidades da operação \\
\hline & Integração com a tecnologia já adotada pela empresa \\
\hline \multirow{3}{*}{ Velocidade operacional } & Aumento da velocidade de recebimento \\
\hline & Aumento da velocidade de separação de pedidos \\
\hline & Aumento na velocidade de identificação de falhas operacionais \\
\hline \multirow{17}{*}{ Controle da operação } & Programação e sequenciamento do recebimento \\
\hline & Controle de portaria e pátio \\
\hline & Erros de conferência no recebimento \\
\hline & Melhor utilização do espaço disponível \\
\hline & Melhor visualização do estoque \\
\hline & Controle de lotes (rastreabilidade, FIFO) \\
\hline & Gerenciamento de devoluções \\
\hline & Programação e sequenciamento da separação de pedidos \\
\hline & Erros na separação do pedido \\
\hline & Erros de conferência no carregamento \\
\hline & $\begin{array}{l}\text { Priorização de atividades (recebimento, armazenagem e/ou } \\
\text { separação de pedidos) }\end{array}$ \\
\hline & Inventário - Acuracidade de estoque \\
\hline & Distribuição e controle da carga de trabalho \\
\hline & Monitoramento do desempenho do colaborador \\
\hline & Monitoramento e controle de produtividade por atividade \\
\hline & Variação do nível de serviço prestado \\
\hline & Variação do nível de estoque \\
\hline
\end{tabular}

Fonte: Elaborado pelos autores (2013).

Essas variáveis foram definidas a partir de informações levantadas no referencial teórico [(BANZATO (2005); BANZATO et al. (2010); BOWERSOX, CLOSS e COOPER (2007), COSTA e GOBBO JUNIOR (2008)] como sendo aspectos relevantes que analisam o processo de implantação do WMS, envolvendo o cenário anterior e posterior à adoção do sistema.

Após a coleta, as informações foram examinadas individualmente em cada estudo de caso e cruzadas, posteriormente, com base na análise de conteúdo, que segundo Bardin (2011, p. 223) "por meio de um sistema de categorias aplica uma teoria (corpo de hipóteses em função de um quadro de referência) ao material". O resultado encontra-se sintetizado nos Quadros 3 e 4 e nas considerações finais. 
Desta forma, a pesquisa passou por seis etapas: (1) definição de objetivos; (2) realização da pesquisa bibliográfica; (3) definição das variáveis de estudo; (4) realização dos estudos de caso; (5) tratamento e análise dos dados; (6) elaboração das considerações finais.

\section{ESTUDOS DE CASO}

A seguir são apresentadas as cinco organizações participantes da pesquisa, subdivididas em duas categorias como exposto acima: empresas desenvolvedoras e usuárias do sitema WMS.

\subsection{Empresas desenvolvedoras do software WMS}

\subsubsection{PC Sistemas}

A PC Sistemas tem como foco de negócio o desenvolvimento de sistemas para o aprimoramento da operação logística na cadeia de suprimentos. Fundada há 26 anos em Belo Horizonte (MG), a empresas expandiu sua operação e possui atualmente unidades em Goiânia, São Paulo, Rio de Janeiro, Joinville, Porto Alegre, Fortaleza, Recife e Belém onde trabalham aproximadamente 500 colaboradores.

$\mathrm{O}$ reconhecimento no mercado aumentou paralelamente à propagação de unidades e hoje atende a cerca de $34 \%$ dos maiores atacadistas e distribuidores do Brasil.

A PC Sistemas desenvolve e comercializa o seu ERP, denominado WinThor, ferramenta de gestão que contribui para o aprimoramento operacional em seguimentos como o atacado/distribuidor e a indústria. Além das aplicações básicas de gestão para as áreas da administração (marketing, comercial e produção), o software também disponibiliza, dentre outros módulos, o WMS.

Com base em entrevista realizada com o responsável pelo desenvolvimento do WMS, percebeu-se que boa parte dos usuários do sistema não tem um conhecimento profundo da ferramenta antes da implantação. De acordo com este estudo de caso, estima-se que dentre os recursos oferecidos pelo sistema (Quadro 
2), a maior parte dos clientes utiliza no máximo 50\%, sendo que, dos 150 clientes atualmente usuários do WinThor não mais do que 10 utilizam acima de $50 \%$ dos recursos do software.

Entretanto, para que a empresa usuária do WMS possa da melhor forma possível realizar a implantação do sistema, o entrevistado destaca a importância de que haja uma preparação da cultura organizacional, explicando detalhadamente o objetivo final e as dificuldades a serem superadas, além da necessidade de que haja colaboração entre todos os processos da organização.

Finalizando a fase pré-implantação, é preciso que o cadastro de produtos e endereços seja feito de forma assertiva, pois em caso de equívocos, poderão ser identificados problemas no controle da operação posteriormente. Salienta-se ainda a importância de que sejam realizados treinamentos antes, durante e após a implantação do sistema. As tendências relacionadas ao aprimoramento do software são mencionadas no Quadro 3.

\subsubsection{Sythex}

Há 19 anos no mercado, a Sythex é uma empresa desenvolvedora de softwares de gestão, cujo foco principal é o WMS. Com duas unidades, em Ribeirão Preto (SP) e em São Paulo capital possui aproximadamente 70 colaboradores e tem como principais clientes empresas como Braspress (maior transportadora de encomendas da América Latina), Netshoes, Hyundai e FEMSA (indústria do grupo Coca-Cola).

Em consonância com o que foi afirmado pela PC Sistema, segundo o responsável pelo desenvolvimento do módulo WMS da Sythex, o nível de informação dos clientes que buscam a ferramenta é geralmente baixo - normalmente conhecem o conceito básico, mas ainda não compreendem em profundidade as funcionalidades e os benefícios potenciais.

Como fatores necessários para que o processo de implantação seja bem sucedido, a empresa salienta o apoio da direção, a preparação e mudança cultural dos colaboradores envolvidos, bem como a realização e checagem do cadastro de produtos, embalagens e endereços a fim de garantir a precisão das informações - a 
principal razão de suspensões e/ou atrasos na conclusão da implantação do WMS, um fator crítico, portanto, para o sucesso do projeto.

Superadas as barreiras iniciais do processo de introdução do sistema, a empresa usuária passa a ter acesso às funcionalidades que auxiliam na execução da rotina diária de trabalho (Quadro 2) e pode obter o benefício de ter uma operação mais eficiente, na qual são minimizadas as ocorrências de falhas e ociosidade, enquanto há uma otimização da velocidade e do controle operacional. As tendências para o aprimoramento do WMS descritas pela Sythex são resumidas no Quadro 3.

\subsection{Empresas usuárias do WMS}

\subsubsection{Distribuidora $X$}

A matriz da Distribuidora X está em Ribeirão Preto (SP), onde atuam 150 colaboradores (20 na área de logística), os quais responsáveis pela movimentação de aproximadamente 1.200 SKUs, envolvendo produtos como macarrão, biscoitos, produtos de higiene pessoal, chá, amendoim e paçoca.

Para gerir suas operações, a empresa usa o WMS, fornecido pela empresa Sythex, cujo projeto de implantação iniciou-se em novembro de 2011. No decorrer deste processo, de acordo com o gerente de logística da empresa, a principal dificuldade encontrada foi a questão da cultura organizacional, identificada na aversão dos colaboradores à nova forma de trabalho imposta pelo software, mesmo tendo recebido treinamento prévio.

Foram necessários, portanto, seis meses de adaptação e, após este período, a empresa identificou alguns benefícios, como a melhor organização do estoque, o controle e a rastreabilidade de lotes (FIFO), o aprimoramento do controle de devoluções, bem como a minimização de erros operacionais e melhor acuracidade de estoque. Todos esses fatores somados contribuíram de forma significativa para a melhoria do nível de serviço prestado ao cliente.

Um ponto relevante a ser destacado é a mudança no processo de separação, anteriormente realizado de forma precária através da visualização pelo separador. Com a utilização do WMS, o tempo de procura foi reduzido drasticamente 
(aproximadamente $80 \%$ ), já que o sistema indica a exata localização dos produtos a serem segregados.

Outra melhoria foi o controle de produtos vencidos no estoque, já que, diariamente, o WMS gera uma lista de itens críticos cujo prazo de validade está próximo. Tal listagem é analisada pela área comercial que negocia previamente com os clientes, de forma a escoar boa parte destes itens. O prejuízo mensal com a perda de produtos vencidos foi reduzido em $86,7 \%$, correspondendo atualmente a uma perda de $\mathrm{R} \$ 2.000,00$ ao mês.

Além das características descritas, identificou-se neste estudo de caso um benefício não mencionado na literatura consultada, a satisfação dos próprios colaboradores em trabalhar de forma mais organizada.

\subsubsection{Atacado $Y$}

$\mathrm{O}$ atacado $\mathrm{Y}$ faz parte de uma rede de lojas em atuação no mercado nacional há 50 anos e presente em 21 estados. Foi definido por conveniência o estudo e análise da filial Ribeirão Preto (SP), que iniciou as suas atividades em 2010 e fatura hoje aproximadamente 168 milhões de reais ao ano, traduzindo-se assim como uma empresa de grande porte. Além disso, em decorrência da dificuldade enfrentada por outras filiais quanto ao controle de estoque (diferenças da ordem de até $R \$$ $400.000,00)$, decidiu-se que esta unidade seria a primeira da rede a implantar o sistema de gestão WMS.

$\mathrm{Na}$ unidade, o atacado trabalha com cerca de 2.500 SKUs, classificados em alimentos, bebidas, limpeza e confinados. A operação usa a armazenagem vertical por meio da estrutura porta-palets, disponibilizando 4.956 posições para estocagem e a movimentação interna é realizada por 50 colaboradores e quatro empilhadeiras.

No início da operação, segundo o analista sênior da área de gestão da armazenagem, a maioria desconhecia a ferramenta e apenas alguns funcionários tinham um conhecimento superficial sobre o WMS. Para auxiliar na execução do projeto, um profissional de São Paulo foi enviado para compartilhar o conhecimento teórico do sistema de gestão e treinar os colaboradores. 
Neste processo, a maior dificuldade encontrada foi a disciplina na execução das atividades, já que a rotina de trabalho do WMS tem como premissa a não realização de movimentações indevidas, ou seja, não autorizada pelo sistema. Fazer com que todos os colaboradores realizassem estritamente as ordens provenientes do sistema foi, portanto, o maior desafio a ser superado.

Foi atribuído então a cada colaborador envolvido nas atividades operacionais o seu próprio coletor de código de barras, o que possibilita o monitoramento da produtividade individual dos funcionários através da comparação entre volume de trabalho previsto e o realizado.

A partir deste acompanhamento, a empresa conseguiu obter um aumento do controle operacional, minimizando o problema inicial enfrentado por outras unidades (a baixa acuracidade de estoque). A filial Ribeirão Preto chega a atingir atualmente o índice de $96,4 \%$ de acuracidade.

\subsubsection{Companhia de Bebidas Ipiranga}

Há 65 anos no mercado, a Cia de Bebidas Ipiranga é uma das maiores franquias do grupo Coca-Cola no Brasil. Sediada em Ribeirão Preto (SP), a indústria atualmente vende seus produtos a mais de 25.000 clientes, distribuídos em 131 municípios, oferecendo 340 SKUs.

A operação logística desta demanda distribui-se em cinco unidades, localizadas em Araraquara, Franca, São João da Boa Vista, Mococa e São Sebastião do Paraíso. Já na logística interna da matriz, objeto de estudo deste artigo, atuam aproximadamente 200 funcionários e são utilizadas 35 empilhadeiras e 30 coletores de código de barras.

Neste contexto, conforme entrevistas realizadas com dois analistas sênior atuantes na gestão da armazenagem da empresa, foram identificadas como as principais razões para a adoção do sistema o alto volume de erros operacionais e a consequente baixa acuracidade de estoque. Por este motivo, em 2008 iniciou-se a implantação do sistema WMS, projeto com duração de seis meses.

$\mathrm{Na}$ fase de pré-implantação, a maioria dos colaboradores não conhecia o conceito do Warehouse Management System, portanto foram realizados 
treinamentos com os funcionários envolvidos na operação. Apesar desta orientação prévia, a adaptação dos colaboradores à forma de trabalho proposta pelo WMS foi a maior dificuldade enfrentada.

A fim de minimizar o surgimento de dúvidas operacionacais após a implantação do sistema e favorecer a adaptação, a empresa treinou uma equipe multiplicadora do conhecimento, composta por pelo menos um funcionário ocupante de cada cargo relevante para a operação logística (conferente, operador de empilhadeira e estoquista).

Segundo a empresa, identificavam-se no inventário diferenças (falta ou sobra) de palets completos em diversos produtos e, após a implantação do sistema, tal divergência resumiu-se a algumas caixas, refletindo em $99,97 \%$ de acuracidade de estoque.

Pode-se afirmar que a causa raiz para esta robusta redução do principal problema que a empresa tinha na sua gestão da armazenagem foi o maior controle operacional propiciado pelo WMS. Através dos relatórios fornecidos pelo sistema, foi possível minimizar os erros de separação e conferência (tanto no recebimento como na expedição), aprimorar a rastreabilidade dos produtos, o controle de estoque e de devoluções.

\section{RESULTADOS}

\subsection{Análise dos desenvolvedores do software WMS}

Com base nos discursos da PC Sistemas e da Sythex, constatou-se que, para ambas, o perfil do cliente que busca a implantação do WMS envolve empresas que detêm certo conhecimento prévio da ferramenta, sem profundidade contudo, situação consonante ao pensamento de Banzato et al. (2010), já mencionado neste artigo.

Por este motivo, observa-se que há indícios de que o fato de a maior parte das empresas não utilizar todo o potencial oferecido pelo sistema está vinculado a esta questão, além do comodismo em situações em que o cliente implanta o WMS, elimina algumas dificuldades previamente identificadas em sua rotina de trabalho, 
mas não explora as demais possibilidades de otimização operacional oferecidas pelo software.

Após a análise das informações provenientes da PC Sistemas e Sythex, foi elaborado o quadro a seguir, que resume das funcionalidades por elas disponibilizadas ao mercado. Estas são segregadas em quadro categorias correspondentes às etapas do processo de armazenagem.

Quadro 2 - Funcionalidades do WMS (PC Sistemas e Sythex)

\begin{tabular}{|c|c|}
\hline Processo & Funcionalidades \\
\hline \multirow{7}{*}{ Recebimento } & Agendamento do recebimento \\
\hline & Captação de notas fiscais dos fornecedores via EDI \\
\hline & Cálculo dos recursos necessários para o recebimento \\
\hline & Gestão de portaria \\
\hline & Convocação ativa para recebimento \\
\hline & Priorização de desembarque \\
\hline & Controle de divergências no recebimento \\
\hline \multirow{11}{*}{ Armazenagem } & Análise da fragmentação dos espaços \\
\hline & Sugestão de reorganização de estoque \\
\hline & Gestão de lotes \\
\hline & Gestão de Shelf-Life \\
\hline & Classificação do status do item \\
\hline & Convocação ativa para armazenagem \\
\hline & Setorização dos locais de armazenagem \\
\hline & Cadastramento das restrições físicas de armazenagem \\
\hline & Endereçamento automático pela Curva ABC \\
\hline & Reabastecimento automático do picking \\
\hline & Realização de inventários cíclicos e estáticos \\
\hline \multirow{7}{*}{$\begin{array}{l}\text { Separação de } \\
\text { Pedidos }\end{array}$} & Convocação ativa para separação e conferência \\
\hline & Ondas de separação parametrizada por regras pré-definidas \\
\hline & Cálculo dos recursos necessários para a separação de pedidos \\
\hline & Picking, priorizando clientes e/ou rotas \\
\hline & Definição de percurso lógico para separação \\
\hline & Controle de separação em tempo real \\
\hline & Conferência dupla de expedição \\
\hline \multirow{16}{*}{$\begin{array}{l}\text { Relatórios } \\
\text { Gerenciais }\end{array}$} & Ocupação do armazém \\
\hline & Visualização gráfica do armazém em 3D online \\
\hline & Detalhamento do inventário \\
\hline & Registro de entradas e saídas por produto, endereço e operador \\
\hline & Resumo de todas as operações (geral, recebimento, armazenagem e expedição) \\
\hline & Análise do desempenho da operação de recebimento \\
\hline & Análise do desempenho da operação de separação de pedidos \\
\hline & Análise do desempenho no atendimento dos pedidos \\
\hline & Análise por produto, volume, transação, período e funcionário \\
\hline & $\begin{array}{l}\text { Parametrização dos tempos médios por tarefa, considerando distância a ser percorrida, } \\
\text { tipo de equipamento utilizado e família de produto movimentada }\end{array}$ \\
\hline & $\begin{array}{l}\text { Registro do tempo gasto em tarefas, tempo ocioso aguardando convocação e tempo } \\
\text { decorrido para aceitar convocação }\end{array}$ \\
\hline & Análise de produtividade por operador, equipe, tarefa ou turno \\
\hline & Planejamento de carga de trabalho \\
\hline & Rastreabilidade das tarefa \\
\hline & $\begin{array}{l}\text { Definição de atividades prioritárias por operador, tipo de equipamento utilizado, área de } \\
\text { atuação e família de produto }\end{array}$ \\
\hline & Distribuição automática de tarefas pendentes \\
\hline
\end{tabular}

Fonte: Elaborado pelos autores (2013).

Revista Produção Online, Florianópolis, SC, v.14, n. 1, p. 195-218, jan./mar. 2014. 
Nesta análise, também foram identificados os benefícios que a ferramenta oferece, as dificuldades existentes para a implantação do sistema e as tendências para o seu aprimoramento, conforme o seguinte quadro resume.

Quadro 3 - Resumo da análise dos desenvolvedores do software WMS.

\begin{tabular}{|c|c|c|}
\hline Variáveis & PC Sistemas & Sythex \\
\hline \multirow{5}{*}{$\begin{array}{l}\text { Dificuldades na } \\
\text { Implementação }\end{array}$} & $\begin{array}{l}\text { Investimento inicial (empresas de } \\
\text { pequeno porte) }\end{array}$ & $\begin{array}{l}\text { Investimento inicial (empresas de } \\
\text { pequeno porte) }\end{array}$ \\
\hline & Preparação Cultural & Mudança Cultural \\
\hline & Apoio da diretoria & Apoio da diretoria \\
\hline & $\begin{array}{l}\text { Mapeamento dos processos } \\
\text { organizacionais }\end{array}$ & $\begin{array}{l}\text { Definição dos processos de } \\
\text { negócio }\end{array}$ \\
\hline & Cadastramento de informações & Cadastramento de informações \\
\hline \multirow{9}{*}{ Benefícios } & $\begin{array}{l}\text { Recebimento mais rápido e } \\
\text { assertivo }\end{array}$ & Recebimento eficiente \\
\hline & $\begin{array}{l}\text { Maior controle e agilidade na } \\
\text { armazenagem }\end{array}$ & $\begin{array}{l}\text { Maior controle da operação de } \\
\text { armazenagem }\end{array}$ \\
\hline & $\begin{array}{l}\text { Separação de pedidos mais rápida } \\
\text { e assertiva }\end{array}$ & $\begin{array}{l}\text { Maior velocidade e minimização } \\
\text { dos erros na separação de pedidos }\end{array}$ \\
\hline & Inventários mais precisos & Melhor acuracidade de estoque \\
\hline & Minimização da ociosidade & Redução da ociosidade operacional \\
\hline & $\begin{array}{l}\text { Redução da ocorrência de erros } \\
\text { operacionais }\end{array}$ & Minimização de erros operacionais \\
\hline & Melhoria do nível de serviço & Aprimoramento do nível de serviço \\
\hline & $\begin{array}{l}\text { Tomada de decisão baseada em } \\
\text { fatos }\end{array}$ & Relatórios de suporte gerencial \\
\hline & $\begin{array}{l}\text { Acompanhamento da operação } \\
\text { online e em tempo real }\end{array}$ & $\begin{array}{l}\text { Acompanhamento da operação } \\
\text { online e em tempo real }\end{array}$ \\
\hline \multirow{3}{*}{ Tendências para o WMS } & $\begin{array}{l}\text { Desenvolvimento de novos } \\
\text { indicadores de desempenho }\end{array}$ & $\begin{array}{l}\text { Desenvolvimento de relatórios } \\
\text { personalizados }\end{array}$ \\
\hline & $\begin{array}{l}\text { Mobilidade (vinculação à } \\
\text { smartphones e tablets) }\end{array}$ & Adaptação à tecnologia RFID \\
\hline & Adaptação à tecnologia RFID & \\
\hline
\end{tabular}

Fonte: Elaborado pelos autores (2013).

É importante salientar que, um fator crítico de sucesso para a implantação do WMS identificado nas entrevistas, mas não mencionado na literatura consultada, é a capacidade da empresa ter os seus processos organizados e mapeados, além de um cadastro de produtos sem erros de digitação ou duplicidade de informação.

Por fim, como tendências ao aprimoramento do sistema WMS, vale ressaltar a necessidade de aprimorar e aprofundar os estudos sobre a tecnologia RFID, ainda pouco utilizada também no Brasil, a fim de que ela possa ser difundida (TWIST, 2004).

Revista Produção Online, Florianópolis, SC, v.14, n. 1, p. 195-218, jan./mar. 2014. 


\subsection{Análise das empresas usuárias do sistema WMS}

Esta etapa consiste no cruzamento dos dados obtidos nas entrevistas realizadas com a Distribuidora $X$, Atacado $Y$ e Cia de Bebidas Ipiranga, abordando de forma sintetizada as variáveis propostas para análise de conteúdo nos procedimentos metodológicos e identificando o cenário pré e pós-implantação do WMS.

Quadro 4 - Resumo da análise das empresas usuárias do WMS

\begin{tabular}{|c|c|c|c|c|}
\hline Indicador & Variável & $\begin{array}{c}\text { Distribuidora } \\
X\end{array}$ & Atacado Y & $\begin{array}{c}\text { Cia de Bebidas } \\
\text { Ipiranga }\end{array}$ \\
\hline \multirow{4}{*}{$\begin{array}{l}\text { Gestão de } \\
\text { Pessoas }\end{array}$} & $\begin{array}{l}\text { Conhecimento do conceito WMS antes } \\
\text { da implantação }\end{array}$ & Não & Não & Não \\
\hline & $\begin{array}{l}\text { Preparação e treinamento pré- } \\
\text { implantação do WMS }\end{array}$ & Sim & Sim & Sim \\
\hline & $\begin{array}{l}\text { Mapeamento e alinhamento dos } \\
\text { processos organizacionais }\end{array}$ & Sim & Sim & Sim \\
\hline & Apoio da alta gerência & Sim & Sim & Sim \\
\hline \multirow{3}{*}{$\begin{array}{c}\text { Tecnologia } \\
\text { da } \\
\text { Informação }\end{array}$} & $\begin{array}{l}\text { Adequação às necessidades da } \\
\text { operação }\end{array}$ & Sim & Sim & Sim \\
\hline & $\begin{array}{l}\text { Integração com a tecnologia já adotada } \\
\text { pela empresa }\end{array}$ & Sim & Sim & Sim \\
\hline & Integração com outras empresas & Não & Não & Não \\
\hline \multirow{3}{*}{$\begin{array}{l}\text { Velocidade } \\
\text { operacional }\end{array}$} & Aumento da velocidade de recebimento & Sim & Sim & Não \\
\hline & $\begin{array}{l}\text { Aumento da velocidade de separação de } \\
\text { pedidos }\end{array}$ & Sim & Sim & Não \\
\hline & $\begin{array}{l}\text { Aumento na velocidade de identificação } \\
\text { de falhas }\end{array}$ & Sim & Sim & Sim \\
\hline \multirow{17}{*}{$\begin{array}{l}\text { Controle da } \\
\text { operação }\end{array}$} & $\begin{array}{l}\text { Programação e sequenciamento do } \\
\text { recebimento }\end{array}$ & Não utilizado & Não utilizado & Não utilizado \\
\hline & Controle de portaria e pátio & Não utilizado & Não utilizado & Não utilizado \\
\hline & Erros de conferência no recebimento & Minimizados & Minimizados & Minimizados \\
\hline & Melhor utilização do espaço disponível & Sim & $\operatorname{Sim}$ & Sim \\
\hline & Melhor visualização do estoque & Sim & Sim & Sim \\
\hline & Controle de lotes (rastreabilidade, FIFO) & Utilizado & Utilizado & Utilizado \\
\hline & Gerenciamento de devoluções & Utilizado & Utilizado & Utilizado \\
\hline & $\begin{array}{l}\text { Programação e sequenciamento da } \\
\text { separação de pedidos }\end{array}$ & Utilizado & Utilizado & Utilizado \\
\hline & Erros na separação do pedido & Minimizados & Minimizados & Minimizados \\
\hline & Erros de conferência no carregamento & Minimizados & Minimizados & Minimizados \\
\hline & Priorização de atividades & Não utilizado & Não utilizado & Não utilizado \\
\hline & Inventário - Acuracidade de estoque & Não utilizado & Utilizado & Utilizado \\
\hline & $\begin{array}{l}\text { Distribuição e controle da carga de } \\
\text { trabalho }\end{array}$ & Não utilizado & Não utilizado & Não utilizado \\
\hline & $\begin{array}{l}\text { Monitoramento do desempenho do } \\
\text { colaborador }\end{array}$ & Não utilizado & Utilizado & Não utilizado \\
\hline & $\begin{array}{l}\text { Monitoramento e controle de } \\
\text { produtividade por atividade operacional }\end{array}$ & Não utilizado & Utilizado & Não utilizado \\
\hline & Variação do nível de serviço prestado & Otimizado & Otimizado & Otimizado \\
\hline & Variação do nível de estoque & Não & Não & Não \\
\hline
\end{tabular}

Fonte: Elaborado pelos autores (2013).

Apenas na distribuidora $X$ e no atacado $Y$ houve aumento na velocidade de execução das atividades rotineiras. Como em ambos os casos até a implantação do

Revista Produção Online, Florianópolis, SC, v.14, n. 1, p. 195-218, jan./mar. 2014. 
WMS as operações de recebimento e separação de pedidos eram realizadas manualmente, percebeu-se um incremento significativo na produtividade. Este cenário não se repetiu na Cia de Bebidas Ipiranga, pois a empresa já utilizava coletores de código de barras antes da implantação deste projeto.

Entretanto, a característica comum identificada nestas empresas, e também o principal benefício destacado por elas, foi o drástico aumento na capacidade de monitoramento e controle do processo de armazenagem, o que aumentou a velocidade na identificação de falhas.

Ainda neste contexto, pode-se afirmar que os três objetos de estudo, através do WMS, foram capazes de melhorar a ocupação do armazém (já que passaram a visualizar melhor o estoque), rastrear o fluxo de produtos e diminuir os erros operacionais, oferecendo melhores níveis de serviço.

Nas três organizações, o endereçamento automático, controle de devolução, rastreabilidade e controle dos lotes, realização do inventário e acompanhamento da operação do armazém são algumas das funcionalidades do WMS mais utilizadas que contribuíram com a otimização dos processos.

Mesmo com tal aprimoramento operacional, algumas das funcionalidades oferecidas pelo sistema não são atualmente utilizadas por estas empresas. A principal razão constatada nas entrevistas realizadas, mas não mencionada pela literatura consultada, é a indisponibilidade de pessoal qualificado em quantidade suficiente para a utilização do WMS de forma adequada. Segundo as organizações usuárias entrevistadas, este é o principal motivo pelo qual a maior parte das empresas, apesar de terem adquirido o software, não exploram todo o seu potencial.

Além disso, outro motivo que leva à não utilização plena do WMS é a existência de algumas funcionalidades que não são aplicáveis a todos os tipos de operação, como é o caso do cross-docking, que não faz parte da rotina de trabalho da Cia de Bebidas Ipiranga, por exemplo.

\section{CONSIDERAÇÕES FINAIS}

Como proposto, este artigo objetivou analisar o sistema WMS identificando as principais barreiras inerentes ao seu processo de implantação, os benefícios e 
funcionalidades atualmente oferecidos ao mercado, bem como as tendências para o aprimoramento da ferramenta.

Com base na análise de conteúdo das informações obtidas a partir do estudo multicasos foi possível elaborar algumas considerações, que expomos a seguir. Primeiramente, quanto às barreiras para a implantação do WMS, houve dois pontos de vistas distintos - o dos desenvolvedores, destacando a importância da realização de um bom cadastro de produtos e o mapeamento dos processos organizacionais por parte das empresas usuárias como fatores determinantes para o sucesso do projeto de implantação do sistema (fatores confirmados pelos usuários); e, por outro lado, a visão das empresas usuárias, que salientaram a relevância do treinamento prévio dos colaboradores envolvidos na operação, além da criação de uma equipe multiplicadora do conhecimento sobre o WMS.

Quanto às funcionalidades atualmente oferecidas ao mercado pelos desenvolvedores analisados (Quadro 2), conforme identificado nas entrevistas realizadas, o usuário tem condições de obter por meio delas como principais benefícios o drástico aumento do controle operacional e a redução de falhas, minimizando custos desnecessários e oferecendo um melhor nível de serviço ao cliente final.

Contribuindo para a geração de uma maior eficiência operacional, foram identificadas (nas empresas desenvolvedoras e nos usuários do sistema) como tendências para o aprimoramento do WMS o desenvolvimento de relatórios customizados e de mais fácil visualização e análise para a tomada de decisão, bem como a integração com a tecnologia RFID e a mobilidade (vinculação a smartphones e tablets).

A elaboração deste artigo científico destaca a identificação de dificuldades, benefícios, funcionalidades e tendências de aprimoramento da ferramenta não mencionados na literatura consultada como possíveis contribuições positivas àquelas empresas que tenham acesso a este estudo.

Como limitação da pesquisa, salienta-se o tamanho da amostra e sugere-se que sejam realizados estudos futuros de caráter quantitativo, envolvendo um número maior de empresas para que os indícios aqui identificados possam ou não ser confirmados.

Revista Produção Online, Florianópolis, SC, v.14, n. 1, p. 195-218, jan./mar. 2014. 


\section{REFERÊNCIAS}

ACCORSI, R.; MANZINI, R.; BORTOLINI, M. A hierarchical procedure for storage allocation and assignment within an order-picking system. A case of study.

International Journal of Logistics Research and Applications. Bologna: v. 15, n. 6, p. 351-364, 2012.

ALVES, P. L. Implantação de tecnologias de automação de depósitos: um estudo de caso. 2000. 132 f. Tese (Doutorado em Administração) - Universidade Federal do Rio de Janeiro: Rio de Janeiro, 2000.

ANDRADE, M. M. Como preparar trabalhos para cursos de pós-graduação. São Paulo: Atlas, 2004.

ANG, M.; LIM, Y.; SIM, M. Robust Storage Assignment in Unit-Load Warehouse. Management Science. Hong Kong, v. 58, n. 11, p. 2114-2130, 2012.

BAKER, P.; CANESSA, M. Warehouse design: a structured approach. European Journal of Operational Research, Bedford: n.193, p. 425-436, 2009.

BALLOU, R. Gerenciamento da cadeia de suprimentos/logística empresarial. 5. ed. Porto Alegre: Bookman, 2006.

BANZATO, E. Tecnologia da informação aplicada à logística. São Paulo: IMAM, 2005.

BANZATO, E. et al. Atualidades na armazenagem. 3. ed. São Paulo: IMAM, 2010.

BARDIN, L. Análise de conteúdo. São Paulo: Edições 70, 2011.

BOWERSOX, D. J.; CLOSS, D. J.; COOPER, M. B. Gestão da cadeia de suprimentos e logística. 2. ed. Rio de Janeiro: Elsevier, 2007.

CHEN, L.; LANGEVIN, A.; RIOPEL, D. The storage location assignment and interleaving problem in an automated storage/retrieval system with shared storage. International Journal of Production Research. London: v. 48, n. 4, p. 991-1011, 2011.

CHIANG, D.; LIN, C;. CHEN, M. The adaptive approach for storage assigment by mining data of warehouse management system for distribution centers. Enterprise Information System. Taiwan: v. 5, n. 2, p. 219-234, 2011.

CHIEN-MING, C. ; GONG, Y.; KOSTER, B. ; NUNEN, J. A flexible evaluative framework for order picking systems. Production and Operations Management, Rotterdam: v. 19, n. 1, p. 70-82, 2010. 
COSTA, W. A.; GOBBO JUNIOR, J. A. Etapas de implementação de WMS: estudo de caso em um varejista moveleiro. GEPROS: Gestão da Produção, Operações e Sistemas: Bauru, v. 4, n. 4, p. 101-121, 2008.

DIAS, S. L. V.; CAULLIRAUX, H. M.; ANTUNES JUNIOR, J. A. V.; LACERDA, D. P. Alinhamento entre sistemas de produção, custo e indicadores de desempenho: um estudo de caso. Produção Online, Florianópolis: v. 7, n. 2, p. 144-169, 2007.

DINTER, B.; LAHRMANN, G.; WINTER, R. Information Logistics as a Conceptual Foundation for Entreprise-Wide Decision Support. Journal of Decision Systems. St. Gallen: v. 19, n. 2, p. 175-200, 2012.

FLEURY, P. F.; WANKE, P.; FIGUEIREDO K. F. Logística empresarial: a perspectiva brasileira. São Paulo: Atlas, 2000.

FREITAS, L.; DINIZ, A.; LEITE, M.; VILLAR, A. Os reflexos do efeito chicote nos custos logísticos de estoque: o caso de uma empresa componente da cadeia de suprimentos de bebidas. Produção Online, Florianópolis: v. 10, n. 2, p. 342367,2010 .

GAMBERINI, R.; GRASSI, A.; MORA, C.; RIMINI, B. An innovative aproach for optimizing warehouse capacity utilization. International Journal of Logistics Research and Applications. Reggio Emilia: v. 11, n. 2, p. 137-165, 2008.

GU, J.; GOETSCHALCKX, M.; MCGINNIS, L. F. Research on warehouse operation: a comprehensive review. European Journal of Operational Research, Atlanta: $n$. 177, p. 1-21, 2007.

JOHNSON, A.; MCGINNIS, L. Performance measurement in the warehousing industry. IIE Transactions. London: v. 43, n. 3, p. 220-230, 2010.

KOSTER, R.; LE-DUC, T.; ROODBERGEN, K. J. Design and control of warehouse order picking: a literature review. European Journal of Operational Research, Rotterdan: v. 1, n. 182, p. 481-501, 2007.

KUTANOGLU, E.; LOHIYA, D. Integrated inventory and transportation mode selection: a service parts logistics system. Transportation Research, Austin: v. 1, n. 44, p. 665-683, 2007.

LOEBBECKE, C.; POWELL, P. Competitive advantege from IT in logistics: the integrated transport tracking system. International Journal of Information Management, Koeln: v. 18, n. 1, p. 17-27, 1998.

LUDWIG, T. D.; GOOMAS, D. T. Performance, accuracy, data delivery, and feedback methods in order selection: a comparison of voice, handheld, and paper technologies. Jornal of Organizational Behavior Management, London: v. 27, n. 1 p. $37-41,2008$. 
MADADI, A.; KURZ, M. E.; ASHAYERI, J. Multi-level inventory management decisions with transportation cost consideration. Transportation Research, Tilburg: v. 46, n. 1, p. 719-734, 2009.

OLIVEIRA NETO, A. A. Metodologia da pesquisa científica. 3. ed. Florianópolis: Visual Books, 2008.

PICK, V. ; DIESEL, L. ; SELLITO, M. Influência dos sistemas de informação na gestão de estoques em pequenos e médios supermercados. Produção Online, Florianópolis: v. 11, n. 2, p. 319-343, 2011.

PONTES, H. L. J; PALMA, J. G.; PORTO, A. J. V. SIMULINVE - Um simulador de inventário para um centro de distribuição de peças. Produção Online, Florianópolis: v. 8, n. 3, p. 1-23, 2008.

REGGIANI, G.; DALCOL, P. Uso da tecnologia da informação na indústria de alimentos e bebidas: um modelo de planejamento para pequenas e médias empresas de manufatura. Produção Online, Florianópolis: v. 11, n. 4, p. 892-920, 2011.

RICHARDSON, R. J. Pesquisa social: métodos e técnicas. 3. ed. São Paulo: Atlas, 1999.

RODRIGUES, E. F. Logística integrada aplicada a um centro de distribuição: comparativo do desempenho do processo de armazenagem após a implementação de um sistema de gerenciamento de armazém (WMS). In SIMPÓSIO DE EXCELÊNCIA EM GESTÃO E TECNOLOGIA, 8., 2011, Resende. Anais... Resende: AEDB, 2011. p. 1-14.

ROODBERGEN, K.; VIS, I. A survey of literature on automated storage and retrieval systems. European Journal of Operational Research. Amsterdam: v. 194, n. 1, p. 343-362, 2009.

RUTNER, S. M.; GIBSON, B. J.; WILLIAMS, S. R. The impacts of the integrated logistics system on electronic commerce and enterprise resource planning system. Transportation Research, Fayetteville: v. 39, n. 1, p. 83-93, 2003.

SAHIN, E.; DALLERY, Y. Assessing the impact of inventory inaccuracies within a Newsvendor framework. European Journal of Operational Research, ChatenaiMalabry: v. 197, n. 1, p. 108-118, 2009.

SALGADO JUNIOR, A.; SEGATTO, M.; CALIA, R.; FIOROTTO, J.; BERTON, L. A tecnologia da informação como suporte ao ajuste da previsão de demanda: um estudo de caso em uma empresa de bebidas carbonatadas. Produção Online, Florianópolis: v. 10, n. 3, p. 621-648, 2010.

SANDERS, N. R.; PREMUS, R. IT applications in supply chain organizations: a link between competitive priorities and organizational benefits. Journal of Business Logistics, Chicago: v. 23, n. 1, p. 65-83, 2002. 
SHIH, S. C.; HSU, S. H. Y.; ZHU, Z.; BALASUBRAMANIAN, S. K. Knowledge sharing - a key hole in the downstream supply chain. Information \& Management, Namdong: v. 49, n. 1, p. 70-80, 2012.

SOUZA, D.; KLIEMANN NETO, F.; ANZANELLO, M. Avaliação de desempenho da cadeia de suprimentos balizada por critérios de competitividade empresarial.

Produção Online, Florianópolis: v. 12, n. 3, p. 756-778, 2012.

TRKMAN, P.; MCCORMACK, K.; OLIVEIRA, M.; LADEIRA., M. The impact of business analysis on supply chain performance. Decision Support Systems. Ljubjana: v. 49, n. 3, p. 318-327, 2010.

TSENG, M. L.; WU, K. J.; NGUYEN, T. T. Information technology in supply chain management: a case of study. Procedia - Social and Behavioral Sciences, London: n. 300, p. 257-272, 2011.

TWIST, D. C. The impact of radio frequency identification on supply chain facilities. Journal of Facilities Management, lowa: v. 3, n. 3, p. 226-239, 2004.

VAIDYANATHAN, G.; DEVARAJ, S. The role of quality in e-procurement performance: an empirical analysis. Journal of Operations Management. South Bend: v. 26, n. 1, p. 407-425, 2008.

VIJAYASARATHY, L. R. An investigation of moderators of the link between technology use in supply chain and supply chain performance. Information and Management, Fort Collins: v. 47, n. 1, p. 364-371, 2010.

YAO, Y.; DRESNER, M. The inventory value of information sharing, continuous replenishment, and vendor-managed inventory. Transportation Research, Bethlehem: v. 44, n. 1, p. 361-378, 2008.

YIN, R. K. Estudo de caso: planejamento e métodos. 4. ed. São Paulo: Bookman: 2009.

YU, M. ; KOSTER, R. Enhancing performance in order picking processes by dynamic storage system. International Journal of Production Research. London: v. 48, n. 16, p. 4785-4806, 2010.

ZHOU, H.; BENTON JR, W. Supply chain practice and information sharing. Journal of Operations Management. Durham: v. 25, n. 1, p. 1348-1365, 2007.

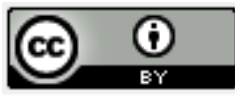

Artigo recebido em 01/05/2013 e aceito para publicação em 09/09/2013. DOI: http://dx.doi.org/10.14488/1676-1901.v14.i1.1582 